\title{
Diagnostic performance of a combination of Mini-Mental State Examination and Clock Drawing Test in detecting Alzheimer's disease
}

This article was published in the following Dove Press journal:

Neuropsychiatric Disease and Treatment

29 April 2013

Number of times this article has been viewed

\section{Yuka Kato' \\ Jin Narumoto' \\ Teruyuki Matsuoka' \\ Aiko Okamura' \\ Hiroyuki Koumi² \\ Yusuke Kishikawa ${ }^{3}$ \\ Shigenori Terashima ${ }^{4}$ \\ Kenji Fukui' \\ 'Department of Psychiatry, Graduate School of Medical Science, Kyoto Prefectural University of Medicine, Kyoto, Japan; ${ }^{2}$ Department of Clinical Psychology, Faculty of Social Welfare, Hanazono University, Kyoto, Japan; ${ }_{3}^{3}$ Misatopia Ogura Hospital, Nagano, Japan; ${ }^{4}$ Graduate School of Psychology, Kansai University, Osaka, Japan}

Correspondence: Yuka Kato Department of Psychiatry, Graduate School of Medical Science, Kyoto Prefectural University of Medicine, 465 Kajii-cho, Kawaramachi-Hirokoji, Kamigyo-ku, Kyoto 602-8566, Japan

Tel +81752515612

Fax+8I 75 25I 5839

Email y-kato@koto.kpu-m.ac.jp
Objective: Because of the growing need for quick cognitive screening tests to distinguish Alzheimer's disease (AD) from mild cognitive impairment (MCI), we compare the diagnostic performance of a combination of the Mini-Mental State Examination (MMSE) and a Clock Drawing Test (CDT) to the Japanese version of the Alzheimer's Disease Assessment Scalecognitive subscale (ADAS-J $\operatorname{cog}$ ) in differentiating between patients with $\mathrm{AD}$, patients with MCI, and healthy controls (HC).

Methods: Data from 146 subjects with AD and 60 subjects with MCI, as well as $49 \mathrm{HC}$, was retrospectively analyzed. We used logistic regression analysis with diagnosis as dependent variables and scores of the MMSE, the CDT-command, and the CDT-copy as independent variables, and receiver operating characteristic analysis to distinguish patients with $\mathrm{AD}$ from patients with MCI or HC

Results: When patients with $\mathrm{AD}$ were compared to $\mathrm{HC}$, the independent predictors of $\mathrm{AD}$ were scores on the MMSE and the CDT-command. This combination was more sensitive than the MMSE alone and has nearly the same sensitivity and specificity as the ADAS-J cog. When patients with $\mathrm{AD}$ were compared to patients with $\mathrm{MCI}$, the independent predictors were the MMSE and the CDT-copy. This combination was more sensitive and specific than the MMSE alone and was almost as sensitive and specific as the ADAS-J cog.

Conclusion: The combination of the MMSE and the CDT could be a powerful screening tool for differentiating between patients with AD, patients with MCI, and HC. Its sensitivity and specificity are comparable to ADAS-J cog, which takes more time.

Keywords: diagnostic techniques, Alzheimer's disease, mild cognitive impairment, Clock Drawing Test, Mini-Mental State Examination

\section{Introduction}

Following the introduction of the concept of mild cognitive impairment (MCI), ${ }^{1}$ general practitioners are frequently required to follow patients diagnosed with $\mathrm{MCI}$ and decide whether patients convert from MCI to Alzheimer's disease (AD). This distinction is essential to starting treatment, including the prescription of cholinesterase inhibitors, in a timely fashion. Considering that MCI has been defined as presence of cognitive impairment among intact daily functions, accurate assessment of functional status is important to detect the conversion from MCI to AD. Although performance-based assessments are ideal measures for functional status, they are difficult to administer in busy primary care settings. Instead, cognitive tests that are quick and easy to administer are feasible for general practitioners.

Although several screening instruments are available for detecting dementia, the Mini-Mental State Examination (MMSE) and the Clock Drawing Test (CDT) have 
been widely used, particularly as quick cognitive screens for dementia in the primary care setting. ${ }^{2}$ Both the MMSE and the CDT are reported to be sensitive in identifying cognitive impairments. A recent meta-analysis of 34 dementia studies and five MCI studies suggested MMSE had a modest sensitivity of $77 \%$ and a specificity of $90 \%$ for application in high-prevalence specialist settings and a sensitivity of $81 \%$ and a specificity of $87 \%{ }^{2}$ Although CDT varies in scoring, a comprehensive literature review suggested that all CDT scorings are remarkably consistent, and both sensitivity and specificity levels have a mean of $85 \%{ }^{3}$ However, the MMSE, in combination with the CDT, has an improved diagnostic performance (sensitivity, 82\%-100\%; specificity, $83.9 \%-95.4 \%$ ), and it is recommended as an easily administered and accurate instrument. ${ }^{4-7}$ Together, these two assessments take about 10 minutes to perform.

In contrast, the Japanese version of the AD Assessment Scale cognitive subscale (ADAS-J cog) is predominantly used in randomized controlled trials that examine the symptomatic benefits of therapy for AD. ${ }^{8,9}$ The ADAS-J $\operatorname{cog}$ consists of eleven tasks measuring the disturbances of memory, language, praxis, attention, and other cognitive abilities, which are often referred to as the core symptoms of AD. It is useful as an instrument for evaluating dementia in a specialized psychogeriatric outpatient setting. However, it takes more than 30 minutes to perform and is thus difficult to use when time is limited.

In this study, we sought to compare diagnostic performance of a combination of the MMSE and the CDT with the ADAS-J cog differentiating between patients with AD, patients with MCI, and healthy controls (HC). The goal was to clarify whether this combination would lead to higher or comparable accuracy than the ADAS-J cog.

\section{Methods}

\section{Subjects}

Subjects with MCI $(n=60)$ and AD $(n=146)$ were recruited from patients in the Aino Hospital. The diagnosis of MCI was made according to Petersen et al's criteria, ${ }^{1}$ which corresponds to amnestic MCI by reported memory concerns, memory impairment on standard tests, absence of significant impairment in activities of daily living, and the absence of dementia. The diagnosis of AD was made according to the National Institute of Neurological and Communicative Disease and Stroke-Alzheimer's Disease and Related Disorders Association's (NINCDS-ADRDA) criteria for probable $A D,{ }^{10}$ respectively. All subjects had been assessed comprehensively by a geriatric psychiatrist, received physical and neurological examinations, and reported their medical histories. Clinical data including functional status was confirmed by family members or other available caregivers. All pertinent laboratory tests and magnetic resonance imagings (MRIs) were reviewed.

$\mathrm{HC}(\mathrm{n}=49)$ were individuals living in the community who participated in the dementia prevention program provided by Aino Hospital. Exclusion criteria were a score of less than 25 on the MMSE, ${ }^{11}$ and a score of less than 6 on the ADAS-J cog. ${ }^{9}$ An interviewer had available access to the social workers in charge of each community before starting the dementia prevention program provided by Aino Hospital to confirm that $\mathrm{HC}$ led an independent life, with both basic and instrumental intact daily living activities.

This study was conducted at Aino Hospital and approved by the hospital's institutional review board. Informed consent was obtained from all subjects or from their substitute decision makers after receiving a complete description of the study.

\section{Instruments}

Subjects were interviewed by trained clinical psychologists with the CDT, the MMSE, and the ADAS-J cog.

\section{CDT}

For the CDT, subjects were presented with a blank sheet of paper and given the following instructions: "I would like you to draw a clock, put in all numbers, and set the hands for 10 past 11 " (CDT-command). Next, they were presented with a drawing of a clock and asked to copy it (CDT-copy). The creators of the instrument, Rouleau et al, ${ }^{12}$ scored subjects on a 10-point scale that is designed to assess the accuracy of the clock face representation (maximum 2 points), the layout of the numbers (maximum 4 points), and the position of the hands (maximum 4 points) independently.

\section{MMSE}

The MMSE ${ }^{11}$ is a frequently used screening instrument in the evaluation of cognitive impairment. It consists of 30 points grouped into seven categories: orientation, registration, attention, calculation, recall, language, and visual construction.

\section{ADAS-J cog}

The ADAS-J $\operatorname{cog}^{9}$ is generally considered the gold standard by regulatory authorities for assessing cognitive function in AD drug trials. It consists of 70 points, with higher scores indicating greater cognitive impairment, which are grouped into eleven categories: word recall, naming, commands, constructional praxis, ideational praxis, orientation, word 
recognition, spoken language, comprehension, word finding, and remembering instructions.

\section{Statistical analysis}

Gender was compared using the Chi-square test. Age and scores of the MMSE, the CDT-command, the CDT-copy, and ADAS-J cog between patients with $\mathrm{AD}$, patients with $\mathrm{MCI}$, and $\mathrm{HC}$ were compared using one-way analysis of variance.

An analysis of covariance was used to compare scores from the MMSE, the CDT-command, the CDT-copy, and ADAS-J cog between patients with AD, patients with MCI, and $\mathrm{HC}$, with age and gender as the covariates. Post hoc testing was performed using Tukey's honestly significant difference tests. Logistic regression analysis was used to optimize the classification performance of MMSE, CDTcommand, and CDT-copy scores, namely, with diagnosis as the dependent variable and scores of the MMSE, the CDT-command, and the CDT-copy as the independent variables. Receiver operating characteristic analysis was used to compare diagnostic performance of the optimized combination with ADAS-J cog scores. Data was analyzed using the Statistical Package for the Social Sciences 17.0 (SPSS; IBM Corporation, Armonk, NY, USA), and resultant statistics where $P<0.05$ were considered significant.

\section{Results}

\section{Subjects' characteristics}

The demographic characteristics with respect to age and gender for each group, along with MMSE, CDT-command, CDT-copy, and ADAS-J cog mean scores, are shown in Table 1. Mean age was significantly lower in $\mathrm{HC}$ as compared to patients with MCI and AD.

\section{Diagnostic performance}

The mean scores in all four neuropsychological tests (MMSE, CDT-command, CDT-copy, and ADAS-J cog) were significantly worse in the AD group as compared to the MCI group and HC (Table 1). After adjusting for the confounding variables of age and gender, AD patients had significantly worse performance in all neuropsychological tests (Table 2).

When the AD group was compared to the HC group, the areas under the curve (AUC) were 0.988 (confidence interval $[\mathrm{CI}]=0.976-0.999)$ for $\operatorname{MMSE}(P<0.001)$, $0.912(\mathrm{CI}=0.871-0.952)$ for CDT-command $(P<0.001)$, $0.764(\mathrm{CI}=0.700-0.829)$ for CDT-copy $(P<0.001)$, and $0.989(\mathrm{CI}=0.979-1.000)$ for ADAS-J $\operatorname{cog}(P<0.001)$. MMSE and CDT-command as identified by logistic
Table I Mean (SD) values for selected clinical variables by group

\begin{tabular}{|c|c|c|c|}
\hline & $\begin{array}{l}\text { HC } \\
(n=49)\end{array}$ & $\begin{array}{l}\mathrm{MCl} \\
(n=60)\end{array}$ & $\begin{array}{l}\text { AD } \\
(n=I 46)\end{array}$ \\
\hline Gender (male/female) & $17 / 32$ & $16 / 44$ & $39 / 107$ \\
\hline Age (years) & $66.1(8.0)$ & $76.1(9.2)^{\mathrm{a}}$ & $79.1(8.0)^{\mathrm{a}, \mathrm{c}}$ \\
\hline MMSE (score out of 30 ) & $29.1(1.2)$ & $24.6(4.1)^{\mathrm{a}}$ & I9.I $(5.1)^{\mathrm{a}, \mathrm{b}}$ \\
\hline $\begin{array}{l}\text { ADAS-J cog } \\
\text { (score out of } 70 \text { ) }\end{array}$ & $3.5(1.6)$ & $10.7(6.1)^{\mathrm{a}}$ & $19.2(8.1)^{\mathrm{a}, \mathrm{b}}$ \\
\hline $\begin{array}{l}\text { CDT-command } \\
\text { (score out of } 10 \text { ) }\end{array}$ & $9.5(0.9)$ & $7.6(2.7)^{\mathrm{a}}$ & $5.6(2.7)^{a, b}$ \\
\hline $\begin{array}{l}\text { CDT-copy } \\
\text { (score out of } 10 \text { ) }\end{array}$ & $10.0(0.2)$ & $9.7(0.7)$ & $8.2(2.3)^{\mathrm{a}, \mathrm{b}}$ \\
\hline
\end{tabular}

Notes: ${ }^{a} P<0.001$ versus $\mathrm{HC}$; ${ }^{\mathrm{p} P}<0.001$ versus $\mathrm{MCl}$; ${ }^{\mathrm{c} P}<0.05$ versus $\mathrm{HC}$; ${ }_{\mathrm{d}} \mathrm{P}<0.05$ versus $\mathrm{MCl}$.

Abbreviations: SD, standard deviation; $\mathrm{HC}$, healthy controls; $\mathrm{n}$, number; $\mathrm{MCl}$, mild cognitive impairment; AD, Alzheimer's disease; MMSE, Mini-Mental State Exam; ADAS-J cog, Japanese version of the Alzheimer's Disease Assessment Scale-cognitive subscale; CDT, clock drawing test.

regression analysis led to slightly higher values than MMSE alone, and values were nearly the same as those obtained with ADAS-J cog alone $(0.997$ [CI] $=0.987-1.000, P<0.001)$, yielding a sensitivity of $91.1 \%$ and a specificity of $100.0 \%$. The positive predictive value was $100.0 \%$ with the prevalence of $\mathrm{AD}$ at $74.9 \%$ in the sample (Table 3 and Figure 1). The equation used in logistic regression for differentiating between patients with $\mathrm{AD}$ and $\mathrm{HC}$ was:

$$
52.67-1.49 \times \text { MMSE }-1.39 \times \text { CDT-command. }
$$

The AUC against the AD group and the MCI group were $0.795(\mathrm{CI}=0.730-0.860)$ for $\operatorname{MMSE}(P<0.001)$, $0.711(\mathrm{CI}=0.630-0.792)$ for CDT-command $(P<0.001)$, $0.686(\mathrm{CI}=0.614-0.759)$ for CDT-copy $(P<0.001)$, and 0.806 (CI $=0.741-0.871)$ for ADAS-J $\operatorname{cog}(P<0.001)$. The best predictors of AD identified by logistic regression analysis were MMSE and CDT-copy. This combination led to slightly higher AUC than MMSE and was nearly the same as that obtained with ADAS-J $\operatorname{cog}(0.811[\mathrm{CI}]=0.747-0.875$, $P<0.001)$, yielding a sensitivity of $75.3 \%$ and a specificity

Table 2 Mean (SD) ANCOVA values

\begin{tabular}{llll}
\hline \multicolumn{1}{c}{$\begin{array}{l}\text { HC } \\
(\mathbf{n}=\mathbf{4 9})\end{array}$} & $\begin{array}{l}\text { MCI } \\
(\mathbf{n = 6 0 )}\end{array}$ & $\begin{array}{l}\text { AD } \\
(\mathbf{n}=\mathbf{~ I 4 6 )}\end{array}$ \\
\hline Adjusted for age and gender & & \\
MMSE & $28.1(0.7)$ & $24.6(0.5)$ & $19.4(0.4)^{\mathrm{a}, \mathrm{b}}$ \\
ADAS-J cog & $4.8(1.0)$ & $10.7(0.9)^{\mathrm{a}}$ & $18.7(0.6)^{\mathrm{a}, \mathrm{b}}$ \\
CDT-command & $9.0(0.4)$ & $7.6(0.3)^{\mathrm{c}}$ & $5.8(0.2)^{\mathrm{a}, \mathrm{b}}$ \\
CDT-copy & $10.0(0.3)$ & $9.7(0.2)$ & $8.2(0.2)^{\mathrm{a}, \mathrm{b}}$ \\
\hline
\end{tabular}

Notes: ${ }^{\mathrm{a}} \mathrm{P}<0.001$ versus $\mathrm{HC}$; ${ }^{\mathrm{b}} \mathrm{P}<0.00$ I versus $\mathrm{MCl}$; ${ }^{\mathrm{c}} \mathrm{P}<0.05$ versus $\mathrm{HC}$.

Abbreviations: SD, standard deviation; ANCOVA, analysis of covariance; HC, healthy controls; $n$, number; $\mathrm{MCl}$, mild cognitive impairment; $\mathrm{AD}$, Alzheimer's disease; MMSE, Mini-Mental State Exam; ADAS-J cog, Japanese version of the Alzheimer's Disease Assessment Scale-cognitive subscale; CDT, clock drawing test. 
Table 3 Diagnostic performance

\begin{tabular}{|c|c|c|c|c|c|c|}
\hline \multirow[t]{2}{*}{ Variables } & \multirow{2}{*}{$\begin{array}{l}\text { AUC } \\
\text { (with } 95 \% \mathrm{Cl} \text { in parentheses) }\end{array}$} & \multirow[t]{2}{*}{ Cut-off } & \multicolumn{4}{|c|}{ Accuracy } \\
\hline & & & SE & SP & PPV & LR+ \\
\hline \multicolumn{7}{|l|}{$A D$ versus $H C$} \\
\hline MMSE & $0.988(0.976-0.999)^{* * *}$ & $24 / 25$ & 85.6 & 100.0 & 100.0 & - \\
\hline CDT-command & $0.912(0.87 \mathrm{I}-0.952)^{* * *}$ & $8 / 9$ & 82.9 & 87.8 & 95.3 & 6.8 \\
\hline CDT-copy & $0.764(0.700-0.829)^{* * *}$ & 10 & 55.5 & 95.9 & 97.6 & 13.5 \\
\hline ADAS-J cog & $0.989(0.979-1.000) * * *$ & 7.5/7.6 & 95.2 & 100.0 & 100.0 & - \\
\hline MMSE + CDT-command & $0.997(0.987-1.000)^{* * *}$ & 0.39 & 91.1 & 100.0 & 100.0 & - \\
\hline \multicolumn{7}{|l|}{$A D$ versus $M C l$} \\
\hline MMSE & $0.795(0.730-0.860)^{* * *}$ & $23 / 24$ & 74.7 & 68.3 & 85.2 & 2.4 \\
\hline CDT-command & $0.711(0.630-0.792)^{* * *}$ & $8 / 9$ & 74.0 & 58.3 & 81.2 & 1.8 \\
\hline CDT-copy & $0.686(0.6 \mid 4-0.759) * * *$ & 10 & 55.5 & 75.0 & 84.4 & 2.2 \\
\hline ADAS-J cog & $0.806(0.74 \mathrm{I}-0.87 \mathrm{I})^{* * *}$ & $13.5 / 13.6$ & 75.3 & 71.7 & 86.6 & 2.7 \\
\hline MMSE + CDT-copy & $0.811(0.747-0.875)^{* * *}$ & 0.67 & 75.3 & 70.0 & 85.9 & 2.5 \\
\hline \multicolumn{7}{|l|}{$\mathrm{MCl}$ versus $\mathrm{HC}$} \\
\hline MMSE & $0.837(0.76 \mathrm{I}-0.912)^{* * *}$ & $26 / 27$ & 63.3 & 95.9 & 95.0 & 15.4 \\
\hline CDT-command & $0.717(0.622-0.812)^{* * *}$ & $8 / 9$ & 50.0 & 87.8 & 83.4 & 4.1 \\
\hline CDT-copy & $0.606(0.50 \mathrm{I}-0.7 \mathrm{II})$ & - & - & - & - & - \\
\hline ADAS-J cog & $0.893(0.83 I-0.955)^{* * *}$ & $13.5 / 13.6$ & 80.0 & 87.8 & 88.9 & 6.6 \\
\hline
\end{tabular}

Notes: Values are AUC (with $95 \% \mathrm{Cl}$ in parentheses). ***P $<0.001$.

Abbreviations: $A U C$, area under the curve; $C l$, confidence interval; SE, sensitivity; SP, specificity; PPV, positive predictive value; LR+, likelihood positive ratio; AD, Alzheimer's disease; HC, healthy controls; MMSE, Mini-Mental State Exam; CDT, Clock Drawing Test; ADAS-J cog, Japanese version of the Alzheimer's Disease Assessment Scale-cognitive subscale; $\mathrm{MCl}$, mild cognitive impairment.

of $70.0 \%$. The likelihood ratio of a positive test was 2.5 . The positive predictive value was $85.9 \%$ with the prevalence of $\mathrm{AD}$ at $70.9 \%$ in the sample, according to the statistical coefficients shown (Table 3 and Figure 1). The equation used in logistic regression for differentiating between patients with $\mathrm{AD}$ and patients with MCI was:

$$
10.09-0.23 \times \text { MMSE }-0.44 \times \text { CDT-copy }
$$

The AUC in differentiating MCI group from HC group were $0.837(\mathrm{CI}=0.761-0.912)$ for $\operatorname{MMSE}(P<0.001)$, $0.717(\mathrm{CI}=0.622-0.812)$ for $\mathrm{CDT}$-command $(P<0.001)$, and $0.893(\mathrm{CI}=0.831-0.955)$ for ADAS-J $\operatorname{cog}(P<0.001)$ (Table 3). However, neither CDT-copy nor the combination of MMSE and CDT was identified by logistic regression analysis.

\section{Discussion}

We examined the abilities of a combination of the MMSE and the CDT administered according to different procedures against the ADAS-J cog alone to differentiate between patients with $\mathrm{AD}$ or $\mathrm{MCI}$ and $\mathrm{HC}$. In differentiating patients with $\mathrm{AD}$ from $\mathrm{HC}$, the combination of the CDT-command
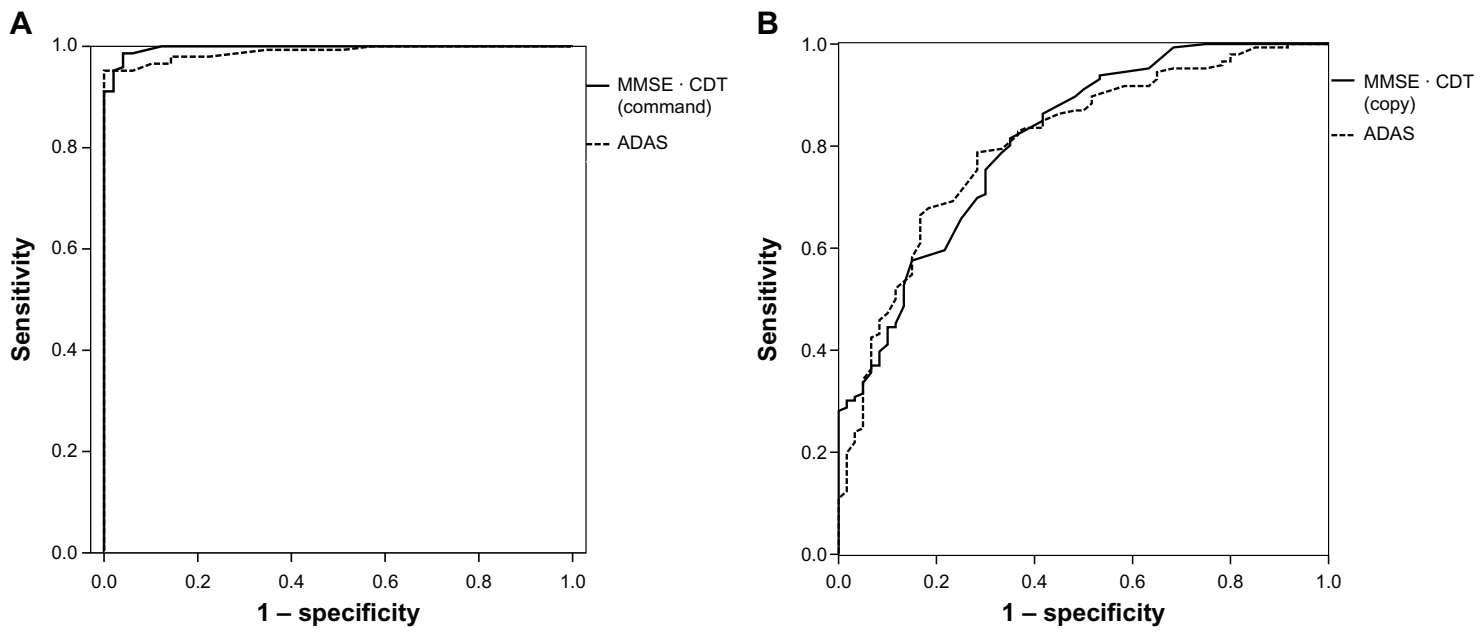

Figure I ROC curves. (A) Subjects with Alzheimer's disease versus healthy controls; (B) subjects with Alzheimer's disease versus mild cognitive impairment. Abbreviation: ROC, receiver operating characteristic. 
and the MMSE had better sensitivity and specificity, while a combination of the CDT-copy and the MMSE had better sensitivity and specificity in differentiating AD from MCI. The screening properties of these combinations were comparable to the ADAS-J cog.

When $\mathrm{AD}$ was compared to $\mathrm{HC}$, the combination of the MMSE and the CDT-command was more sensitive than the MMSE alone, and has nearly the same sensitivity and specificity as the ADAS-J cog. These findings are consistent with previous studies that found enhanced accuracy in the detection of AD with the combined MMSE and CDT, as opposed to the MMSE or the CDT alone. ${ }^{4-7}$ Heinik et $\mathrm{al}^{6}$ showed that combining the MMSE and the CDT improved sensitivity to $100 \%$ and specificity to $91 \%$, as compared with the MMSE alone (sensitivity, 96\%; specificity, 81\%). Further, this combination performed better than the Cognitive and self-contained part of the Cambridge Examination for Mental Disorders of the Elderly, a 30-minute multicognitive task. ${ }^{13}$ We found that the MMSE combined with the CDT-command showed enhanced sensitivity as a first-stage dementia screen, as CDT-command requires multiple cognitive domains, such as receptive language, memory, abstraction, and executive functions. . $^{3,13-15}$

When patients with AD were compared to patients with $\mathrm{MCI}$, the combination of the MMSE and the CDT-copy had better sensitivity and specificity than the MMSE or the CDT alone, and was nearly the same as that obtained with the ADAS-J cog. The CDT-copy mainly requires visuospatial and visuoconstructional function, ${ }^{3,15}$ as with the doublepentagon copy of the MMSE. However, the CDT-copy might be more sensitive for measuring visuospatial and visuoconstructional functions than the double-pentagon copy, because the CDT-copy requires the spatial layout of the complex component features of a clock. In addition, the double-pentagon copy is only worth 1 point on the MMSE, and disability might be underestimated as measured by the total MMSE score. As a screening test, CDT-command is more popular than CDT-copy. However, our results suggest that CDT-copy is useful to differentiate AD from MCI if it is administered with MMSE.

The combination of the MMSE and the CDT was found to have a higher sensitivity than $(89.4 \%)$ and the identical specificity of ( $83.9 \%$ ) the MMSE alone (sensitivity, $86.4 \%$; specificity, 83.9\%) in detecting mild AD and MCI. ${ }^{5}$ From our data, we found that the MMSE has good sensitivity (74.7\%), but it lacks good specificity (68.3\%); conversely, the CDT-copy lacks sensitivity (55.5\%) but has high specificity $(75.0 \%)$ in differentiating MCI from AD. Thus, this combination enhanced accuracy complementarily.
Hence, the combination of MMSE and CDT would be useful as one of the assessments when general practitioners evaluate dementia and decide timing to prescribe a cholinesterase inhibitor in a primary care or community setting.

On the other hand, the combination of the MMSE and the CDT was not able to accurately differentiate patients with MCI from $\mathrm{HC}$ in this study. Cacho et $\mathrm{al}^{5}$ studied 66 individuals with mild AD, 21 individuals with MCI, and $66 \mathrm{HC}$ individuals in a memory clinic, and found that the AUC for this combination (mini-clock) were higher than what was obtained using the MMSE or the CDT alone in differentiating patients with $\mathrm{MCI}$ from $\mathrm{HC}$. Cacho et $\mathrm{al}^{5}$ concluded that mini-clock assessment is reasonably accurate in distinguishing patients with MCI from $\mathrm{HC}$; however, this study had small sample sizes, especially for patients with MCI. Only a few studies have tried to test the screening properties of this combination in differentiating between patients with MCI and $\mathrm{HC}$. Hence, further research is necessary to conclude whether this combination can be used to differentiate patients with MCI from HC.

This study has several limitations. First, the MCI construct has still been controversial, and many types and stages of MCI have been proposed. We diagnosed subjects with MCI solely based on their informant-based functional status. However, some cases might possibly have met criteria for dementia if their functional status had been more strictly assessed. First, we should have defined stages of MCI, early or late MCI, and used the activities of daily living and instrumental activities of daily living to evaluate the functional autonomy of AD, not only MCI. Second, HC were significantly younger as compared to patients with MCI and AD. This may have enhanced sensitivity too much. However, it is noteworthy that cognitive scores for the MMSE, the CDT-command, and the ADAS-J cog were significantly worse in individuals with $\mathrm{AD}$ and $\mathrm{MCI}$ compared to $\mathrm{HC}$, even after adjusting for the confounding variable of age. Third, we did not collect data on the level of education of our subjects. Since HC were sampled from a dementia prevention program, they might be more likely to have higher education levels than the patients. However, all subjects are known to have at least received the Japanese compulsory education of 6 years.

In summary, these results are consistent with previous studies that reported high sensitivity and specificity with the combination of the MMSE and the CDT when AD was compared with $\mathrm{HC}$ and with MCI. Our results also suggest that the combination of the MMSE and the CDT may be a similarly accurate replacement for the ADAS-J cog, which is to be used when screening time is limited. 


\section{Disclosure}

The authors report no conflicts of interest in this work.

\section{References}

1. Petersen RC, Smith GE, Waring SC, Ivnik RJ, Tangalos EG, Kokmen E. Mild cognitive impairment: clinical characterization and outcome. Arch Neur. 1999;56(6):303-308.

2. Mitchell AJ. A meta-analysis of the accuracy of the mini-mental state examination in the detection of dementia and mild cognitive impairment. $J$ Psychiatr Res. 2009;43(4):411-431.

3. Shulman KI. Clock drawing: is it the ideal cognitive screening test? Int $J$ Geriatr Psychiatr. 2000;15(6):548-561.

4. Aprahamian I, Martinelli JE, Cecato J, Yassuda MS. Screening for Alzheimer's disease among illiterate elderly: accuracy analysis for multiple instruments. J Alzheimers Dis. 2011;26(2):221-229.

5. Cacho J, Benito-León J, García-García R, Fernández-Calvo B, VicenteVillardón JL, Mitchell AJ. Does the combination of the MMSE and clock drawing test (mini-clock) improve the detection of mild Alzheimer's disease and mild cognitive impairment? J Alzheimers Dis. 2010;22(3):889-896.

6. Heinik J, Solomesh I, Bleich A, Berkman P. Are the clock drawing test and the MMSE combined interchangeable with CAMCOG as a dementia evaluation instrument in a specialized outpatient setting? J Geriatr Psychiatry Neurol. 2003;16(2):74-79.

7. Schramm U, Berger G, Müller R, Kratzsch T, Peters J, Frölich L. Psychometric properties of clock drawing test and MMSE or Short Performance Test (SKT) in dementia screening in a memory clinic population. Int J Geriatr Psychiatry. 2002;17(3):254-260.
8. Rosen WG, Mohs RC, Davis KL. A new rating scale for Alzheimer's disease. Am J Psychiatry. 1984;141(11):1356-1364.

9. Homma A, Fukuzawa K, Tsukada Y, Ishii T, Hasegawa K, Mohs RC. Development of a Japanese version of Alzheimer's disease assessment scale (ADAS) (in Japanese). Japanese Journal of Geriatric Psychiatry. 1992; 3: 647-655.

10. McKhann G, Drachman D, Folstein M, Katzman R, Price D, Stadlan EM. Clinical diagnosis of Alzheimer's disease: report of the NINCDS-ADRDA Work Group under the auspices of Department of Health and Human Services Task Force on Alzheimer's Disease. Neurology. 1984;34(7):939-944.

11. Folstein MF, Folstein SE, McHugh PR. "Mini-mental state". A practical method for grading the cognitive state of patients for the clinician. J Psychiatr Res. 1975;12(3):189-198.

12. Rouleau I, Salmon DP, Butters N, Kennedy C, McGuire K. Quantitative and qualitative analyses of clock drawings in Alzheimer's and Huntington's disease. Brain Cogn. 1992;18(1):70-87.

13. Roth M, Tym E, Mountjoy CQ, Huppert FA, Hendrie H, Verma S, Goddard R. CAMDEX. A standardised instrument for the diagnosis of mental disorder in the elderly with special reference to the early detection of dementia. Br J Psychiatry. 1986;149:698-709.

14. Royall DR, Mulroy AR, Chiodo LK, Polk MJ. Clock drawing is sensitive to executive control: a comparison of six methods. J Gerontol B Psychol Sci Soc Sci. 1999;54(5):P328-P333.

15. Freedman M, Leach L, Kaplan E, Winocur G, Shulman KI, Delis DC. Clock Drawing: A Neuropsychological Analysis. New York, NY: Oxford University Press; 1994.
Neuropsychiatric Disease and Treatment

\section{Publish your work in this journal}

Neuropsychiatric Disease and Treatment is an international, peerreviewed journal of clinical therapeutics and pharmacology focusing on concise rapid reporting of clinical or pre-clinical studies on a range of neuropsychiatric and neurological disorders. This journal is indexed on PubMed Central, the 'PsycINFO' database and CAS.

\section{Dovepress}

The manuscript management system is completely online and includes a very quick and fair peer-review system, which is all easy to use. Visit http://www.dovepress.com/testimonials.php to read real quotes from published authors. 